\title{
Electrospun nanofiber-based drug delivery systems
}

\author{
Deng-Guang Yu ${ }^{1}$, Li-Min Zhu ${ }^{1^{*}}$, Kenneth White ${ }^{2}$, Chris Branford-White ${ }^{2}$ \\ ${ }^{1}$ College of Chemistry, Chemical Engineering and Biotechnology, Donghua University, Shanghai, China \\ ${ }^{2}$ Institute for Health Research and Policy, London Metropolitan University, London, UK
}

Received 15 May 2009; revised 8 June 2009; accepted 11 June 2009.

\begin{abstract}
Electrospinning is a very simple and versatile process by which polymer nanofibers with diameters ranging from a few nanometers to several micrometers can be produced using an electrostatically driven jet of polymer solution or polymer melt. Significant progress has been made in this process throughout the past few years and electrospinning has advanced its applications in many fields, including pharmaceutics. Electrospun nanofibers show great promise for developing many types of novel drug delivery systems (DDS) due to their special characteristics and the simple but useful and effective top-down fabricating process. The current state of electrospun nanofiber-based DDS is focused on drug-loaded nanofiber preparation from pharmaceutical and biodegradable polymers and different types of DDS. However, there are more opportunities to be exploited from the electrospinning process and the corresponding drug-loaded nanofibers for drug delivery. Additionally, some other related challenges and the possible resolutions are outlined in this review.
\end{abstract}

Keywords: Electrospinning; Nanofibers; Drug Delivery Systems; Controlled Release

\section{INTRODUCTION}

Electrospinning, firstly reported in 1934, has been used for more than 60 years, and yet is under developed in studying the fabrication of continuous nanofibers. The term "electrospinning", derived from "electrostatic spinning", was coined relatively recently. Since 1980s and especially in recent years, the electrospinning process has regained more attention probably due in part to a surging interest in nanotechnology, as ultrafine fibers or fibrous structures of various polymers with diameters in the submicron/nanometer range can be easily fabricated using this process. A survey of open publications and patents related with electrospinning in the past several years is given in Figure 1 The data were obtained from Elsevier ScienceDirect, Wily InterScience and the Dewent Innovations Index, and clearly demonstrates that electrospinning has attracted increasing attention in recent times. [1-3]

A schematic diagram demonstrating the process of electrospinning of polymer nanofibers is shown in Figure 2. There are basically three components: a high voltage supplier, a capillary tube with a pipette or needle of small diameter, and a metal collecting screen. In electrospinning a high voltage is used to create an electrically charged jet of polymer solution or melt out of the pipette. Before reaching the collecting screen, the solution jet evaporates or solidifies, and is collected as an interconnected web of small fibers. One electrode is placed into the spinning solution/melt and the other attached to the collector. In most cases, the collector is simply grounded. The electric field is applied across the end of the capillary tube that contains the solution fluid held by its surface tension. This induces a charge on the surface of the liquid. Mutual charge repulsion and the contraction of the surface charges to the counter electrode create a force directly opposite to the surface tension. As the intensity of the electric field is increased, the hemispherical surface of the fluid at the tip of the capillary tube elongates to form a conical shape known as the Taylor cone. Further increasing the electric field, a critical value is attained with which the repulsive electrostatic force overcomes the surface tension and the charged jet of the fluid is ejected from the tip of the Taylor cone. The discharged polymer solution jet undergoes an instability and elongation process, which allows the jet to become very long and thin. Meanwhile, the solvent evaporates, leaving behind a charged polymer fiber. In the case of the melt the discharged jet solidifies when it travels in the air stream. [2-12]

Electrospinning appears to be affected by the following parameters and variables: 1) system parameters such as molecular weight, molecular weight distribution and architecture (branched, linear, etc.) of the polymer, and polymer solution properties (viscosity, conductivity, dielectric constant, and surface tension, charge carried by the spinning jet) and 2) process parameters such as 


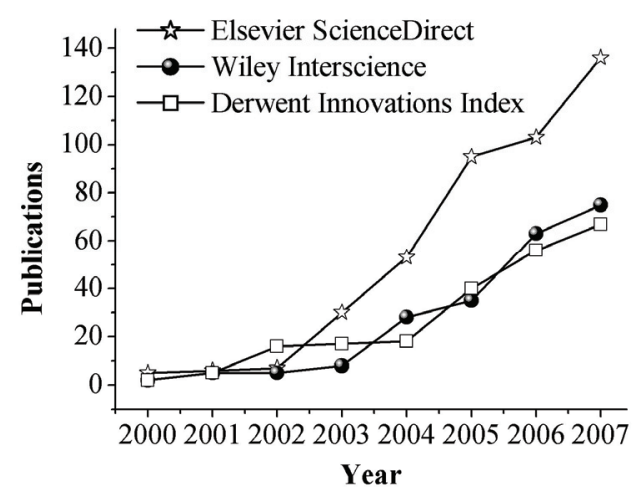

Figure 1. The increase of literature electrospinning from several databases (Search term is "electrospinning" within "source title").

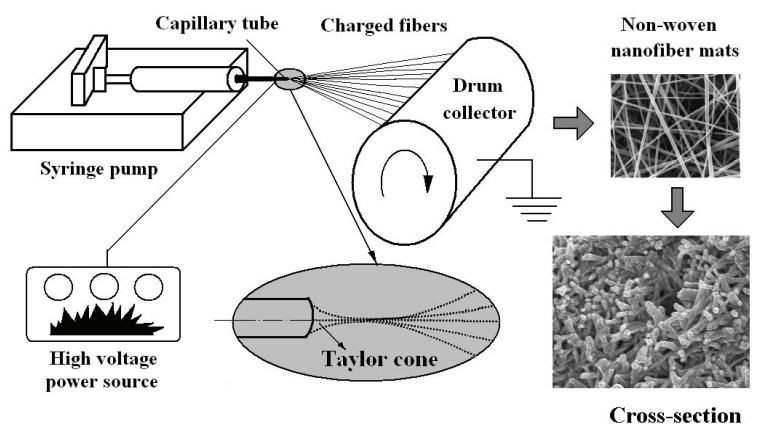

Figure 2. The process of electrospinning.

electric potential, flow rate and concentration, distance between the capillary and collection screen, ambient parameters (temperature, humidity and air velocity in the chamber) and finally motion of the target screen. By appropriately varying one or more of the above parameters, nanofibers can be successfully electrospun from a rich variety of materials that include polymers, biopolymers, DNA, protein, composites, and ceramics and even relatively small macromolecules such as phospholipids. [2-4]

A number of processing techniques such as drawing, template synthesis, phase separation and self-assembly have been used to prepare polymer nanofibers in recent years. However these methods have disadvantages such as: material limitation, they are time-consuming and they require complicated processing systems. As far as electrospinning is concerned it is not only a simple one-step top-down process for fabricating nanofibers, but also the co-processing of polymer mixtures, chemical crosslinking can be carried out that provide a variety of pathways for controlling the chemical composition of the nanofibers. These provide a wide range of properties such as strength, weight, elasticity, porosity and charged surface areas. Moreover electrospinning also provides the capacity to lace together a variety of nanoparticles or nanofillers types that can be encapsulated into a nanofi- ber matrix. Functional micro/nano particles may be dispersed in polymer solutions, which are then electrospun to form composites in the form of continuous nanofibers and nanofibrous assemblies. All these endow electrospinning with outstanding manufacturing capabilities but utilizing an easy process and capable of excellent flexibility. Additionally, electrospinning seems to be the only method that can be further developed for mass production of one-by-one continuous nanofibers from various polymers. [3]

Over the past several decades, polymer sciences have been the backbone of pharmaceutics [13]. Many pharmaceutical polymer excipients are commonly used in the development of novel drug delivery systems (DDS) now. Combined usage of electrospinning with pharmaceutical polymers provides novel strategies for developing novel DDS, and through the manipulation of electrospinning process, may offer flexibility for tailoring DDS's properties.

\section{CHARACTERISTICS OF ELECTROSPUN FIBERS}

Polymer nanofibers have a diameter in the order of a few nanometers to over $1 \mu \mathrm{m}$ (more typically $50 \sim 500 \mathrm{~nm}$ ) and possess unique characteristics, such as: extraordinary high surface area per unit mass (for instance, nanofibers with $\sim 100 \mathrm{~nm}$ diameter have a specific surface of $\sim 1000 \mathrm{~m}^{2} / \mathrm{g}$ ), coupled with remarkably high porosity, excellent structural mechanical properties, high axial strength combined with extreme flexibility, low basis weight, and cost effectiveness are among others.

Another interesting aspect of using nanofibers is that it is feasible to modify not only their morphology and their (internal bulk) content but also the surface structure to carry various functionalities. Nanofibers can be easily post-synthetically functionalized (for example by chemical or physical vapour deposition). Furthermore, it is even feasible to control secondary structures of nanofibers in order to prepare nanofibers with core/sheath structures, nanofibers with hollow interiors and nanofibers with porous structures. [10]

Economically, the electrospinning nano-manufacturing process is relatively low cost compared to that of most bottom-up nanofiber-fabricating methods. The resulting nanofibers are often uniform, continuous and do not require expensive purification protocols. The nanofibers are relatively easy to be scaled up for productivity due to the top-down process and the designing of multiple jets for synchronous electrospinning. [14] Additionally, the nanofibers have one dimension at the microscopic scale but another dimension macroscopically. This unique characteristic endows nanofiber mats with both the merits possessed by functional materials on the nano-meter scale, and these have advantages over conventional solid membrane such as easy processing, ease 
of packaging and shipping.

These outstanding properties make polymer nanofibers as good candidates for many applications. For example nanofibers mats are now being considered for composite materials reinforcement, sensors, filtration, catalysis, protective clothing, biomedical applications (including wound dressing and scaffolds for tissue engineering, implants, membranes and drug delivery systems), space applications such as solar sails, and microand nanooptoelectronics. Thus the properties of nanofibers make them useful for systems for developing nanofibers-based DDS.

\section{CURRENT STATE OF ELECTROSPUN NANOFIBER-BASED DDS}

Research about electrospun nanofibers as drug delivery systems is in the early stage of exploration. [3] Many current researches focus on the preparation and characterization of polymer nanofibers. To date, it is generally believed that nearly one hundred different polymers, mostly dissolved in solvents yet some heated into melts, have been successfully spun into ultrafine fibers. How to transit nanofibers into DDS is creating much attention. It is clear from Figure 3 that the open publications related to electrospun nanofiber-based DDS are increasing more sharply than those related with nanofibers.

The first report about electrospinning fibers as DDS was noted by Kenawy et al. [5] Electrospun fiber mats were explored as drug delivery vehicles using tetracycline hydrochloride as a model drug. The mats were made either from poly (lactic acid) (PLA), poly (ethylene-co-vinyl acetate) (PEVA), or from a 50:50 blend of the two from chloroform solutions. Release profiles showed promising results when they were compared to a commercially available DDS--Actisite ${ }^{\circledR}$ (Alza Corporation, Palo Alto, CA), as well as to the corresponding cast films. An early patent registered by Ignatious and Baldoni described electrospun polymer nanofibers forpharmaceutical compositions, which can be designed to provide rapid, immediate, delayed, or modified dissolution, such as sustained and/or pulsatile release characteristics. [6]

Later studies on the preparation of nanofibers from polymers with different drug-loaded capabilities and the corresponding DDS were reported, such as transdermal, fast dissolving and implantable DDS (Figure 4). Most of the early work focused on the sustained release profiles and all types of active pharmaceutical ingredients were used as model drugs, such as small molecular drug, herbs, proteins, poorly water-soluble and water-soluble drugs, DNA, genes and vaccines. The polymers include biodegradable hydrophilic polymers, hydrophobic polymers and amphiphilic polymers. [3,15,16]

Zhang et al. reported that degradable heparin-loaded poly ( $\varepsilon$-caprolactone) fiber mats were successfully fab- ricated by electrospinning. The highly sulphated heparin hetropolymer remained homogenous in the spinning solution and was evenly distributed throughout the fabricated polymers. A sustained release of heparin could be achieved from the fibers over 14 days with the release diffusionally controlled over this period. The released heparin retained biological properties and functionality. [17] Chew et al. investigated the feasibility of encapsulating human $\beta$-nerve growth factor (NGF) that was stabilized in the carrier protein, bovine serum albumin (BSA) in a copolymer of $\varepsilon$-caprolactone and ethyl ethylene phosphate. Partially aligned protein encapsulated fibers were obtained and the protein was found to be randomly dispersed throughout the electrospun fibrous mesh in an aggregated form. The sustained release of NGF by diffusion was obtained for at least 3 months. PC12 neurite outgrowth assay confirmed that the bioactivity of electrospun NGF was retained throughout the period of sustained release. [18] Luu et al. utilized electrospinning to fabricate synthetic polymer/DNA composite for therapeutic application in gene delivery designed for tissue engineering. The composite was non-woven, nano-fibered, membranous structures composed predominantly of poly(lactide-co-glycolide) (PLGA) random

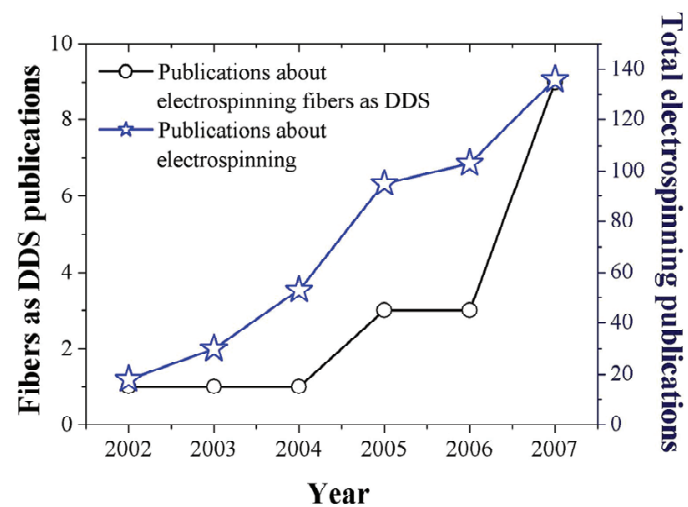

Figure 3. The increase of literature about e-spinning nanofibers as DDS (Search term is "electrospinning" in "title and "drug delivery" in "abstract").

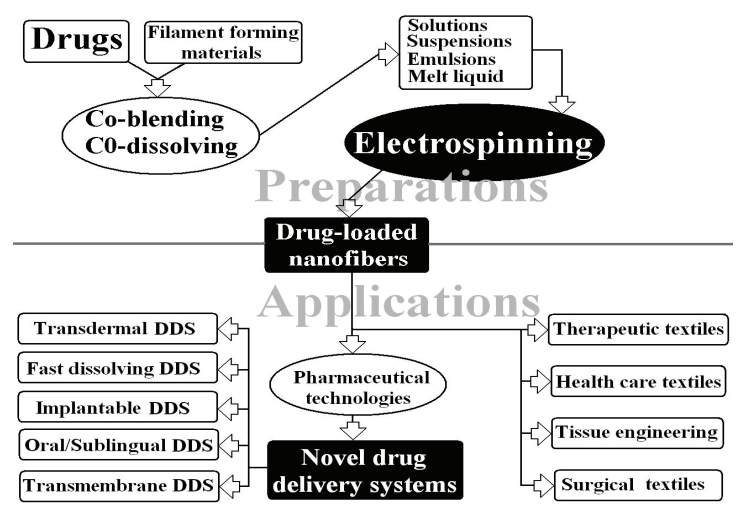

Figure 4. Applications and preparations of electrospun drugloaded nanofibers. 
copolymer and a poly(D,L-lactide)-poly(ethylene glycol) (PLA- PEG) block copolymer. Release of plasmid DNA from the composite was sustained over a 20-day study period, with maximum release occurring at $\sim 2 \mathrm{~h}$. Cumulative release profiles indicated amounts released were approximately $68-80 \%$ of the initially loaded DNA. Results indicated that DNA released directly from these electrospun fibers was indeed intact, capable of cellular transfection, and successfully expressed the encoded protein $\beta$-galactosidase. [19]

Electrospun nanofibers are often used to load insoluble drugs for enhancing their dissolution properties due to their high surface area per unit mass. Tungprapa et al. prepared ultra-fine fiber mats of cellulose acetate (CA) for four different types of model drugs, i.e., naproxen (NAP), indomethacin (IND), ibuprofen (IBU), and sulindac (SUL), from 16\% w/v CA solutions in $2: 1 \mathrm{v} / \mathrm{v}$ acetone/N,N-dimethylacetamide (DMAc) by electrospinning. The amount of the drugs in the solutions was fixed at $20 \mathrm{wt} \%$ based on the weight of CA powder. No drug aggregates were observed on the surfaces of the fibers. The maximum release of the drugs from loaded fiber mats were ranked as follows: NAP $>$ IBU $>$ IND $>$ SUL and this did not correspond to their solubility properties. [7] Taepaiboon et al. reported that the molecular weight of the model drugs played a major role on both the rate and the total amount of drugs released from the prepared drug-loaded electrospun PVA nanofibers. The rate and the total amount of the drugs released decreasing with increasing molecular weight of the encapsulated drugs. [8]

Taepaiboon et al. also reported that mats of PVA nanofibres were successfully prepared by the electrospinning process and were developed as carriers of drugs for a transdermal drug delivery system. Besides the water insoluble drugs naproxen (NAP), and indomethacin (IND), freely water soluble sodium salicylate, was also spun into the PVA fibers. [8] Xu et al. proposed a novel process, i.e., 'emulsion electrospinning' to prepare core-sheath fibers to incorporate a water soluble drug into a hydrophobic or an amphiphilic polymer fiber. [20] Maretschek et al. [21] recently reported the electrospinning of emulsions composed of an organic poly (L-lactide) solution and an aqueous protein solution, which yielded protein containing nanofiber nonwovens having a mean fiber diameter of approximately $350 \mathrm{~nm}$. This provided the opportunity to tailor the release profile of macromolecular active ingredients. All the above reports demonstrated that electrospun drug-loaded nanofibers were able to provide sustained release profiles for different types of active pharmaceutical ingredients.

Studies previously reported the influence of a high electrical potential on the chemical integrity of the drugs, the comparatively controlled release characteristics of nanofibers and the release-controlled mechanisms. Tungprapa et al. [7] and Taepaiboon et al. [8] confirmed that the electrospinning process did not affect the chemical integrity of the drugs by ${ }^{1} \mathrm{H}$-nuclear magnetic resonance. Taepaiboon et al. [8] proved that the drug-loaded electrospun PVA mats exhibited better release characteristics of four model drugs than drugloaded as-cast films and Tungprapa et al. [7] showed that the release of drugs from the CA drug- loaded films was due mainly to the gradual dissolution of aggregates on the film surfaces, whilst the diffusion of the drugs incorporated within the films occurred to a lesser extent. On the contrary, since no presence of the drug aggregates was found on the surface of the drug-loaded CA fibers, the release of the drugs from the drug-loaded fiber mats was mainly by the diffusion of the drugs from the fibers, as the fiber mats could swell appreciably in the testing medium. Moreover the fibrous morphology of the drug-loaded fiber mats after the drug release assay at $24 \mathrm{~h}$ was still intact. Verreck et al. confirmed that the application of electrostatic spinning to pharmaceutical applications resulted in dosage forms with better useful and controllable dissolution properties than the simple physical mixture, solvent cast or melt extruded samples. [22]

Although many types of DDS have been prepared from electrospun drug-loaded nanofibers, no related clinical experiments have been reported and only few in vivo drug delivery researches have been undertaken, which were mainly associated with the cancer research. Ranganath et al. reported the paclitaxel-loaded biodegradable implants in the form of microfiber discs and sheets developed using electrospinning were used to treat malignant glioma in vitro and in vivo. The fibrous matrices not only provided greater surface area to volume ratio for effective drug release rates but also provided needed implantability into the tumor resected cavity of a post-surgical glioma. [23]

The advantages of employing electrospinning technology to prepare DDS are not as yet fully exploited. Nanotechnology is now having an impact in biotechnology, pharmaceutical and medical diagnostics sciences. Nanodrugs are at the forefront of bioengineering for diseases and represent the next generation of medical therapies that will impact worldwide markets and especially the healthcare industry [24]. Furthermore electrospinning as noted before has gained more attention due in part to a surging interest in nanotechnology, as ultrafine fibers or fibrous structures of various polymers with small diameters. [25] On the other hand, electrospinning should exert more influence on new DDS development through providing novel strategies for conceiving and fabricating them. 


\section{NOVEL STRATEGIES PROVIDED BY ELECTROSPINNING FOR NEW DDS}

From the current literature, several advantages of using electrospun polymer nanofibers as DDS are recognized, and these merit further consideration in developing new types of DDS.

Firstly, due to the high surface area to volume ratio, polymer nanofibers provide a useful pathway for delivery of water insoluble drug. With the recent advent of high throughput screening of potential therapeutic agents, the number of poorly soluble drug candidates has risen sharply and the formulation of poorly soluble compounds for oral delivery now presents one of the most frequent and greatest challenges to formulation scientists in the pharmaceutical industry. [26] Solid dispersion is considered to be the most suitable choice to improve dissolution rates and hence the bioavailability of the poorly water soluble drug. [27] However, the practical applicability of solid dispersion systems has remained limited due to difficulties in conventional methods of preparation, poor reproducibility of physiochemical properties, dosage formulation and lack of feasibility for scaling-up manufacturing processes. [28] Electrospun nanofibers may provide novel approaches as to how the dissolution rate of even very poorly soluble compounds might be improved to minimize the limitations of oral availability.

Xie et al. developed electrospun PLGA-based nanofibers as implants for the sustained delivery of anticancer drug to treat C6 glioma cells in vitro. Differential scanning calorimetry (DSC) results suggest that the drug was in the solid solution state in the polymeric micro- and nanofibers. In vitro release profiles suggest that paclitaxel sustained release was achieved for more than 60 days. Cytotoxicity test results suggest that the $\mathrm{IC}_{50}$ value of paclitaxel-loaded PLGA nanofibers is comparable to the commercial paclitaxel formulation-Taxol®. [29]

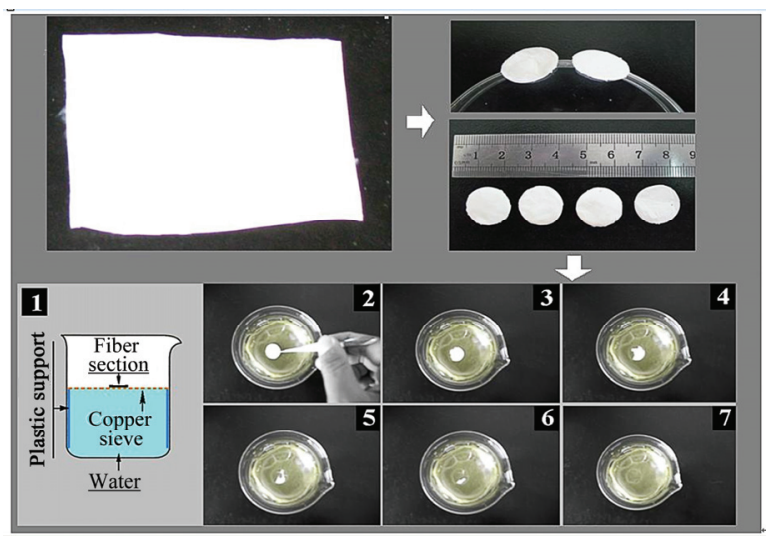

Figure 5. Fast dissolving drug delivery membrane.
Verreck and co-workers assessed the application of water-soluble polymer-based nanofibers prepared by electrostatic spinning as a means of altering the dissolution rate of the poorly water-soluble drug, itraconazole. DSC measurements found that the melting endotherm for itraconazole was not present, suggesting the formation of an amorphous solid dispersion or solution. Dissolution studies assessed several presentations including direct addition of the non-woven fabrics to the dissolution vessels, folding weighed samples of the materials into hard gelatin capsules and placing folded material into a sinker. [22] Studies in our laboratory have been undertaken on the solubility improvement of poorly water-soluble drugs and the corresponding fast dissolving DDS. [30] Shown in Figure 5 is a patent product of a rapid dissolving drug delivery membrane, which can absorb water and dissolve within several seconds a poorly water-soluble drug.

Second, the drug release profile can be easily finely tailored by modulation not only of the composition of the nanofiber mats but also the morphology of nanofibers, the process and the micro-structure. Core-sheath structure is a very useful structure for all kinds of applications. Several fabrication techniques have been proposed to prepare ultrafine fibers configured in a core-sheath structure, such as self-assembly, laser ablation, template synthesis, and a tube by fiber templates process. Core-sheath fibers can be prepared by 'emulsion electrospinning'. Xu et al. [16] reported that uniform core-sheath nanofibers were prepared by electrospinning a water-in-oil emulsion in which the aqueous phase consists of a poly(ethylene oxide) (PEO) solution in water and the oily phase is a chloroform solution of an amphiphilic poly(ethylene glycol)-poly(L-lactic acid) (PEG-PLA) diblock copolymer. The obtained fibers are composed of a PEO core and a PEG-PLA sheath with a sharp boundary in between. By adjusting the emulsion composition and the emulsification parameters, the overall fiber size and the relative diameters of the core and the sheath can be altered. The stretching and evaporation induced de-emulsification and the transformation from the emulsion to the core-sheath fibers.

Concentric electrospinning is a very promising approach to fabricate core-sheath fibers. [31] Coaxial electrospinning (Figure 6) is an alternative approach to encapsulate drugs or biological activties inside polymer nanofibers. In a typical process (Figure 6), two or more polymer liquids are forced by an electrostatic potential to eject out through different but co-axial capillary channels, resulting in a core-shell structured composite nanofiber. As long as the shell fluid is able to be processed along with electrospinning, the core fluid can either be or not be electrospinnable. One advantage in using such a technique is an effective protection of easily denatured biological agents and the potential to wrap all substances 


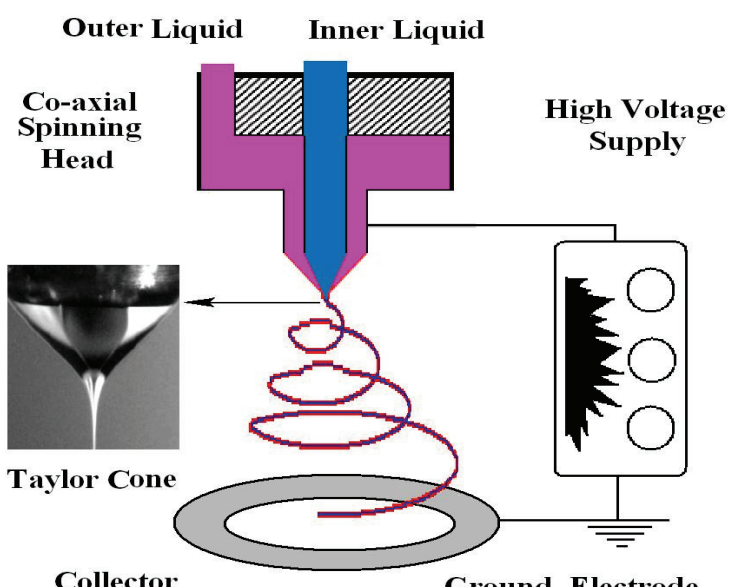

Figure 6. Co-axial electrospinning systems.

in the core regardless of drug-polymer interactions. Hence, drugs, proteins, growth factors, and even genes can be incorporated into nanofibers by dissolving them in the core solutions. [32-34]

Huang et al. used co-axial electrospinning to prepare core-sheath nanofibers for controlled release of multi drugs. Polycaprolactone was used as the shell and two medically pure drugs, Resveratrol and Gentamycin Sulfate, were used as the cores. The drugs were released in a controlled way without any initial burst effect. [32]

Third, there is a lot of flexibility in the use of nanofibers in designing various dosage forms to achieve maximum bioavailability of a drug moiety for different drug delivery routes. Electrospun drug-loaded nanofibers are often used as mid dosage forms. They can be further turned into different kinds of DDS for all types of drug delivery routes, such as for transdermal administration, oral administration, pulmonary administration, subcutaneous implant, or for dissolution into a liquid media for administration, such as a suspension or solution or by parenteral/intramuscular or intracavernosum injection and so on. [35]

Besides preparing DDS solely from electrospun fibers, researchers often combine the electrospinning process with other special substances to prepare DDS. Shalaby describes a partially absorbable, fiber-reinforced composite in the form of a ring, or a suture-like thread, with modified terminals for use as a controlled delivery system of bioactive agents. The composite comprised an absorbable fiber construct capable of providing timedependent mechanical properties of a biostable elastomeric matrix containing an absorbable microparticulate ion-exchanger to modulate the release of the bioactive agents for a desired period of time at a specific biological site, such as the vaginal canal, peritoneal cavity, scrotum, prostate gland, an ear loop or subcutaneous tissue. [36]

Fourth, electrospun nanofibers often have higher drug encapsulation efficiency than other nanotechnologies.
Xie et al. reported that the encapsulation efficiency for paclitaxel-loaded PLGA micro- and nanofibers was more than $90 \%$. The electrospun paclitaxel-loaded biodegradable micro- and nanofibers are promising for the treatment of brain tumour as alternative drug delivery devices. [29] Xu et al. showed that a water-soluble anticancer agent, doxorubicin hydrochloride, was totally encapsulated within the electrospun poly (ethylene glycol)-poly (1-lactic acid) (PEG-PLLA) fibers when its content in the fibers was $5 \mathrm{wt} \%$. [37] Other advantages of drug-loaded nanofibers, such as small diameter of the nanofibers, can provide short diffusion passage length. Also, high surface area facilitates mass transfer and effective drug release.

As mentioned above, the drug-loaded nanofibers derived from electrospinning not only have one dimension at the microscopic scale but another dimension in the macroscopic form. This unique characteristic endows the electrospun drug-loaded nanofibers with both the merits possessed by the DDS on the nano-meter scale in altering the biopharmaceutic and pharmacokinetic properties of the drug molecule for favorable clinical outcomes, and also the advantages of conventional solid dosage forms such as easy processing, good drug stability, and ease of packaging and shipping.

\section{SOME CHALLENGES AND THE POSSIBLE RESOLUTIONS}

Although some reports in the literature have demonstrated that electrospinning is useful for preparing new DDS there are still some challenges associated with the preparation of electrospun nanofiber-based DDS.

Electrospinning is a simple micro-processing technique to make ultrafine or nanometer range fibers generally from high molecular weight polymer solutions or melts. The largest challenge lies firstly in understanding the electrospinning process as a fluid dynamics system. In order to control the properties, geometry, and mass production of the nanofibers, it is necessary to understand quantitatively how electrospinning transforms the fluid solution through a millimeter diameter capillary tube into solid fibers which are four to five orders smaller in diameter. Secondly, the efficiency of electrospinning is still a bottleneck. Studies on multiple nozzles need to be undertaken and these will form a platform for electrospinning industrialization. [38-40]

To date, most of the release tests have been done in vitro. What is more, several problems must be resolved for further applications such as the drug loading, the initial burst effect, the residual organic solvent, the stability of active agents, and the combined usage of new biocompatible polymers. Drug-loading is always a problem for nano DDS. Although drug loading over $50 \%$ of the total weight was reported, the drug loading in the nanofibers still needs to be increased in many cases. The 
reason is that drug often influences the spinnability of the polymer solution. The viscosity range of a polymer solution which is spinnable is about $1-20$ poises and the surface tension between 35 and 55 dynes/cm is suitable for fiber formation. Relatively high drug loading may also easily cause the uneven distribution of the drug in the nanofiber resulting in initial burst effects for electrospinning fibers except for co-axial fibers. [41]

The initial burst effect is a common phenomenon for nano drug delivery systems with high surface area such as nano- or microspheres, liposomes and hydrogels. The reason for this phenomenon has been investigated by a number of laboratories. For ordinary electrospinning, drug-loaded nanofibers electrospun from mixtures of drugs and polymers the drug release characteristics rely on the drug being encapsulated within the nanofibers. However due to surface effects the drug particles in the nanofibers tend to accumulate on the fiber surface. Thus, a burst release at an initial stage is inevitable unless the blend of drug and polymer carrier is fully integrated into the nanofiber at a molecular level. [32]

Zeng et al. studied the encapsulation of the lipophilic drug paclitaxel and the hydrophilic drug doxorubicin hydrochloride in the electrospun PLLA fiber mats and their release kinetics. Preferable encapsulation of paclitaxel was found due to its good compatibility with PLLA and solubility in chloroform/acetone solvent, whereas doxorubicin hydrochloride was observed on or near the surfaces of PLLA fibers. The release results of these drugs confirmed that the release of paclitaxel from electrospun PLLA fiber samples followed nearly zero-order kinetics due to the degradation of the fibers. However a burst release was found for doxorubicin hydrochloride due to the diffusion of the drug on or near the surfaces of the fiber sample. Therefore, the solubility and compatibility of the drugs in the drug/polymer/solvent system were the decisive factors for the preparation of the electrospun fiber formulation with constant release of the drugs. In order to encapsulate a majority of the drugs inside the polymer fibers and thus to acquire a constant and stable drug release profile, a lipophilic polymer should be chosen as the fiber material for a lipophilic drug while a hydrophilic polymer should be employed for a hydrophilic drug and the solvents used should be suitable for both drug and polymer. [41]

To smoothen or even eliminate the initial burst effects, post-treatment methods are often considered. Within this context Kenawy et al. reported that the burst release of ketoprofen was eliminated when the electrospun poly(vinyl alcohol) fiber mats were stabilized against disintegration in water by treatment with methanol. [5] Taepaiboon post-treated electrospun fibre mats of poly(vinyl alcohol) (PVA) containing sodium salicylate by exposing the fibre mats to the vapour from $5.6 \mathrm{M}$ aqueous solution of either glutaraldehyde or glyoxal for various exposure time intervals, followed by a heat treatment in a vacuum oven. With increasing the exposure time in the cross-linking chamber, the morphology of the electrospun fiber mats gradually changed from a porous to a dense structure. Cross-linking appreciably reduced the release of sodium salicylate from the drug-loaded fiber mats and both the rate and the total amount of the drug released decreased functions with exposure time interval in the cross-linking chamber. [42]

Certainly, the core-shell structure fiber with the drug in the core can eliminate the burst effects. Research also showed that surfactants can reduce the surface tensions and the diameter of resulted nanofibers, improve the drug uniformity and thus can smoothen the burst effect. [43]

To adapt the development of pharmaceutics, one of the emphases is the preparation of novel polymers drug-loaded nanofibers, for example, polymer with environmental sensitive characteristics. Chunder et al. reported that ultrathin fibers comprising two oppositely charged weak polyelectrolytes PAA/PAH were fabricated using electrospinning. These fibers are capable of controlling drug releasing through $\mathrm{pH}$ changes. The releasing properties of PAA/PAH fibers was tuned by depositing different coatings onto fiber surfaces. A sustained and a temperature controlled drug releasing in PBS solutions was achieved by depositing perfluorosilane coatings and PAA/PNIPAAM multilayers onto the fiber surfaces, respectively. [44]

In theory, comprehension and clarification of the relationship between the release profiles and the electrospinning parameters help to select suitable materials, optimize electrospinning process, and thus to improve the consistence between design and manufacturing, reduce the time to market for novel DDS. Since the physical form of the active agent in a dosage form can influence the product performance, it is often necessary to quantify the different solid phases in a system for preparing a robust dosage form. In nanofibers, the possible interactions between the drugs and the excipients in the dissolution and electrospinning processes should be thoroughly investigated for further developing novel DDS.

Drug release profiles from the drug delivery systems should be precisely predicted or programmed so that any possibility of dose dumping and subject-to-subject variability can be minimized. The relationships between the drug controlled release profile and the electrospinning parameters should be elucidated. Mathematical models of drug release from nanofibers can be used to elucidate the underlying drug transport mechanism and predict the resulting drug release kinetics as a function of the nanofibers (structure, geometry and composition). In conclusion, there are still many things to do to enable the electrospun nanofiber-based DDS to go into clinical applications. 


\section{ACKNOWLEDGEMENT}

We thank the UK-CHINA Joint Laboratory for Therapeutic Textiles and Biomedical Textile Materials, China Postdoctoral Science Foundation (NO.20080440565), and Grant 08JC1400600 from Science and Technology Commission of Shanghai Municipality for the financial support.

\section{REFERENCES}

[1] Formhals, Process and apparatus for preparing artificial threads, US Pat 1975504, October-02-1934.

[2] Doshi, J. and Reneker, D.H., (1995) Electrospinning process and applications of electrospun fibers, Electrostatics, 35, 151-160.

[3] Huang, Z.M., Zhang, Y.Z., Kotaki, M. and Ramakrishna, S. (2003) A review on polymer nanofibers by electrospinning applications in nanocomposites, Composites Sci. Tech., 63, 2223-2253.

[4] McKee, M.G., Layman, J.M., Cashion, M.P., Timothy, E.L. (2006) Phospholipid nonwoven electrospun membranes, Science, 311, 353-355.

[5] Kenawy, E.R., Bowlin, G.L., Mansfield, K., Layman, J., Simpson, D.G., Sanders, E.H., and Wnek, G.E. (2002) Release of tetracycline hydrochloride from electrospun poly (ethylene-co-vinylacetate), poly(lactic acid), and a blend, J. Control. Release, 81, 57-64.

[6] Ignatious, F., Baldoni, J.M. (2001) Electrospun pharmaceutical compositions, WO Pat 0154667.

[7] Tungprapa, S., Jangchud, I., and Supaphol, P. (2007) Release characteristics of four model drugs from drugloaded electrospun cellulose acetate fiber mats, Polymer, 48, 5030-5041.

[8] Taepaiboon, P., Rungsardthong, U., Supaphol, P. (2006) Drug-loaded electrospun mats of poly(vinyl alcohol) fibres and their release characteristics of four model drugs, Nanotechnology, 17, 2317-2329.

[9] Dzenis, Y. (2004) Spinning continuous fibers for nanotechnology, Science, 304, 1917-1919.

[10] Chronakis, I.S. (2005) Novel nanocomposites and nanoceramics based on polymer nanofibers using electrospinning process-A review, J. Mat. Proc. Tec., 167, 283-293.

[11] Agarwal, S., Wendorff, J.H. and Greiner, A. (2008) Use of electrospinning technique for biomedical applications, Polymer, 49, 5603-5621.

[12] Yu, D.G., Shen, X.X., Branford-White, C., White, K., Zhu, L.M. and Bligh, S.W.A. (2009) Oral fast-dissolving drug delivery membranes prepared from electrospun PVP ultrafine fibers," Nanotechnology, 20, 055104.

[13] Pillai, O. and Panchagnula, R. (2001) Polymers in drug delivery, Curr. Opin. Chem. Biol., 5, 447-451.

[14] Varabhas, J.S., Chase, G.G. and Reneker, D.H. (2008) Electrospun nanofibers from a porous hollow tube, Polymer, 49, 4226-4229.

[15] Shen, X.X., Yu D.G., Ranford-White, C. B. and Zhu, L.M., (2008) Preparation and characterization of ultrafine eudragit L100 fibers via electrospinning, The 3rd International Conference on Bioinformatics and Biomedical Engineering ( $\left(C B B E^{\prime} 09\right)$, May18, Shanghai, China.

[16] Xu, X., Chen, X., Wang, Z. and Jing, X. (2009) Ultrafine PEG-PLA fibers loaded with both paclitaxel and doxorubicin hydrochloride and their in vitro cytotoxicity, Euro. J. Pharm. Biopharm., 72, 18-25.

[17] Zhang, Y.Z., Wang, X., Feng, Y., Li, J., Lim, C.T. and Ramakrishna, S. (2006) Coaxial electrospinning of fluorescein isothiocyanate - conjugated bovine serum albumin)-encapsulated poly( $\varepsilon$-caprolactone) nanofibers for sustained release, Biomacromolecules, 7, 1049-1057.

[18] Chew, S.Y., Wen, J., Yim, E.K.F. and Leong, K.W. (2005) Sustained release of proteins from electrospun biodegradable fibers, Biomacromolecules, 6, 2017-2024.

[19] Luu, Y.K., Kim, K., Hsiao, B.S., Chu, B. and Hadjiargyrou, M. (2003) Development of a nanostructured DNA delivery scaffold via electrospinning of PLGA and PLAPEG block copolymers, J. Control. Release, 89, 341-353.

[20] Xu, X., Zhuang, X., Chen, X., Wang, X., Yang, L. and Jing, X. (2006) Preparation of core-sheath composite nanofibers by emulsion electrospinning, Macromol. Rapid Commun., 27, 1637-1642.

[21] Maretschek, S., Greinerb, A. and Kissel, T. (2008) Electrospun biodegradable nanofiber nonwovens for controlled release of proteins, J. Control. Release, 127, 180187

[22] Verreck, G., Chun, I., Peeters, J., Rosenblatt, J. and Brewster, M. E. (2003) Preparation and characterization of nanofibers containing amorphous drug dispersions generated by electrostatic spinning, Pharm Res, 20, 810-817.

[23] Ranganath, S.H. and Wang, C.H., (2008) Biodegradable microfiber implants delivering paclitaxel for post- surgical chemotherapy against malignant glioma, Biomaterials, 29, 2996-3003.

[24] Devalapally, H., Chakilam, A. and Amiji, M.M. (2007) Role of nanotechnology in pharmaceutical product development, J. Pharm. Sci., 96, 2547-2565.

[25] Rutledge, G.C. and Fridrikh, S.V. (2007) Formation of fibers by electrospinning, Adv. Drug Del. Rev., 59, 13841391.

[26] Leuner, C. and Dressman, J. (2000) Improving drug solubility for oral delivery using solid dispersions, Eur. $J$. Pharm. Biopharm., 50, 47-60.

[27] Chokshi, R.J., Zia, H., Sandhu, H.K., Shah, N.H. and Malick, W.A. (2007) Improving the dissolution rate of poorly water soluble drug by solid dispersion and solid solution-pros and cons, Drug Delivery, 14, 33-45.

[28] Sethia, S. and Squillante, E. (2003) Solid dispersions: Revival with greater possibilities and applications in oral drug delivery, Crit. Rev. Thera. Drug Carrier System, 20, 215-247.

[29] Xie, J. and Wang, C.H. (2006) Electrospun micro- and nanofibers for sustained delivery of paclitaxel to treat C6 glioma in vitro, Pharm. Res., 23, 1817-1826.

[30] Yu, D.G., Branford-White, C., Shen, X.X., Zhang, X.F. and Zhu, L.M., (2010) Solid dispersions of ketoprofen in drug-loaded electrospun nanofiber, Journal of Dispersion Science and Technology, 31, article 8.

[31] McCann, J.T., Li, D. and Xia, Y. (2005) Electrospinning of nanofibers with core-sheath, hollow, or porous structures, J. Mater. Chem., 15, 735-738.

[32] Huang, Z.M., He, C.L., Yang, A., Zhang, Y., Han, X.J., Yin, J. and Wu, Q. (2006) Encapsulating drugs in biodegradable ultrafine fibers through co-axial electrospinning, J. Biom. Mat. Res., 77A, 169-179.

[33] Loscertales, I.G., Barrero, A., Guerrero, I., Cortijo, R., 
Marquez, M. and Ganan-Calvo, A.M. (2002) Micro/nano encapsulation via electrified coaxial liquid jets, Science, 295, 1695-1698.

[34] Li, D. and Xia, Y. (2004) Direct fabrication of composite and ceramic hollow nanofibers by electrospinning, Nano Letter, 4, 933-938.

[35] Ignatious, F. and Baldoni, J.M. (2003) Electrospun pharmaceutical compositions, US Pat 2003017208.

[36] Shalaby, S.W. (2007) Partially absorbable fiber-reinforced composites for controlled drug delivery, $E P$ 1786356.

[37] Xu, X.L., Yang, L.X., Xu, X.Y., Wang, X., Chen, X.S., Liang, Q.Z., Zeng, J. and Jing, X.B. (2005) Ultrafine medicated fibers electrospun from $\mathrm{W} / \mathrm{O}$ emulsions, $J$. Control. Release, 108, 33-42.

[38] Kim, G.H., Cho, Y.S., Kim, W.D. (2006) Stability analysis for multi-jets electrospinning process modified with a cylindrical electrode, Euro. Polym. J., 42(9), 2031-2038.

[39] Yoon, H., Kim, G.H., Kim, W.D. (2008) Electrohydrodynamic process supplemented by multiple-nozzle and auxiliary electrodes for fabricating PCL nanofibers, Polymer, 32(4), 334-339.
[40] Heikkila, P. and Harlin, A. Parameter study of electrospinning of polyamide-6, Euro. Polym. J., 44(10), 30673079.

[41] Zeng, J., Yang, L., Liang, Q., Zhang, X., Guan, H., Xu, X., Chen, X. and Jing, X. (2005) Influence of the drug compatibility with polymer solution on the release kinetics of electrospun fiber formulation, J. Control. Release, 105, 43-51.

[42] Taepaiboon, P., Rungsardthong, U., Supaphol, P. (2007) Effect of cross-linking on properties and release characteristics of sodium salicylate-loaded electrospun poly (vinyl alcohol) fibre mats, Nanotechnology, 18, 175102 (11pp).

[43] Zeng, J., Xu, X.Y., Chen, X.S., Liang, Q.Z., Bian, X.C. and Yang, L.X. (2003) Biodegradable electrospun fibers for drug delivery, J. Control. Release, 92, 227-231.

[44] Chunder, A., Sarkar, S., Yu, Y. and Zhai, L. (2007) Fabrication of ultrathin polyelectrolyte fibers and their controlled release properties, Colloids and Surfaces B: Biointerfaces, 58, 172-179. 